\title{
PERCEPÇÃO OCUPACIONAL: A QUALIDADE DO AR INTERNO EM BIBLIOTECA PÚBLICA, CEARÁ, BRASIL
}

\author{
Lydia dayanne Maia Pantoja ${ }^{1}$, José Capelo Neto $^{1}$, \\ Ronaldo FERreira do NASCIMENTo ${ }^{1}$, AnA BÁRbara de ARAúuo Nunes ${ }^{1}$ \\ ${ }^{1}$ Universidade Federal do Ceará - UFC \\ Programa de Pós-Graduação em Engenharia Civil (Saneamento Ambiental) \\ <lydiapantoja@yahoo.com.br>, <capelo@ufc.br>. \\ <ronaldo@ufc.br>. <abarbara@deha.ufc.br>
}

DOI: $10.21439 /$ conexoes.v10i3.854

\begin{abstract}
Resumo. A má qualidade do ar interno em bibliotecas vem merecendo atenção, devido à quantidade de substratos favoráveis à ação de biodegradadores sobre os acervos escritos/digitais. Embora não seja causa de morte, seus sintomas podem causar desconforto e baixa da produtividade de seus trabalhadores. Nesse ínterim, objetivou-se realizar uma analise frente ao discernimento dos trabalhadores de uma biblioteca pública de referência no munícipio de Fortaleza, Ceará, sobre a qualidade do ar. Entre janeiro a abril/2013 foram aplicados questionários, abordando perfil do trabalhador, atividade laboral, estado de saúde e qualidade do ar do ambiente de trabalho. Foram analisados 38 funcionários, média etária de 42 anos, média de 12 anos de trabalho, alocados em 9 setores. Constatou-se que $89 \%$ classificaram o ar como empoeirado ou com presença de odores, $82 \%$ apresentaram sintomas relacionados à Síndrome dos Edifícios Doentes, bem como, 71\% nunca se queixaram a administração, $100 \%$ nunca ouviram a respeito da SED e 37\% faltaram mais de 9 dias devido a problemas de saúde nos últimos meses. Por fim, aspira-se que a presente pesquisa, ao demonstrar a fragilidade do conhecimento dos trabalhadores, leve a maior divulgação em bibliotecas e, como consequência, melhorias no monitoramento e controle ambiental, permitindo avanços dessa temática no Brasil.
\end{abstract}

Palavras-chaves: Saúde do trabalhador. Qualidade do ar. Biblioteca.

Abstract. The poor air quality in libraries has attracted attention from researchers due to the quantity of substrates favorable to the action of biodegraders on written/digital archives' substrates. Although not a potential cause of death, the symptoms provoked by these organisms can cause discomfort and low productivity of workers. The objective of this study was to discover the opinions of employees of a public library in the municipality of Fortaleza, Ceará regarding the air quality. Between January and April 2013, questionnaires were administered to obtain information on the worker profile, labor activity, health and air quality of the working environment. Thirty-eight employees, with average age of 42 years and an average of 12 years on the job, allocated to nine sectors, were surveyed. It was found that $89 \%$ rated the air as dusty or malodorous, $37 \%$ reported they had missed more than nine days due to health problems in recent months and $90 \%$ reported sick building syndrome symptoms, but $71 \%$ had never complained to management and $100 \%$ had never heard about SBS. This study, by demonstrating the fragile knowledge of library workers, can lead to better disclosure and as a result better environmental.

Keywords: Occupational health. Air quality. Library.

\section{INTRODUÇÃO}

As mudanças arquitetônicas iniciadas nos anos de 1970 nos países desenvolvidos geraram os chamados prédios selados, onde o microclima artificial tornou-se gerador de poluentes do ar interior. Atualmente, estima-se que boa parte da população dos centros urbanos chega a permanecer $90 \%$ de seu tempo diário em locais fechados e climatizados artificialmente, sejam em seus trabalhos, restaurantes, áreas de lazer, escolas, hospitais, biblio- 
tecas, carros e mesmo em seus lares (HUSMAN et al. 2002, MEHTAL et al., 2002, SAUNDERS, 2005). Essa baixa circulação interna do ar expõem as pessoas a poluentes comumente encontrados no ar interno, sendo responsáveis por vários efeitos adversos à saúde.

A Organização Mundial da Saúde reconheceu a existência da baixa qualidade do ar de interiores como uma questão de saúde ocupacional, pois se refere à uma relação de causa e efeito entre as condições ambientais de trabalho e a redução da produtividade do trabalhador decorrente de agressões ao bem-estar e ao conforto, denominando-a de Síndrome do Edifício Doente - SED (WHO, 2006). Esses edifícios possuem 20\% dos usuários expostos com alguns sintomas característicos (ROBERTSON, 1995), como, ataques de falta de ar, tosse, expectoração, aperto no peito, espirros, irritação e secreção nasal, irritação na garganta, irritação ocular, febre, dor muscular, dermatites de contato, entre outros (DEGOBBI; GAMBALE, 2008). Esses sintomas parecem estar vinculados ao tempo de permanência no local, já que diminuem rapidamente ao sair para o almoço e ao retornar para casa (ROCHA, 2008). Com a SED, os locais de trabalho se tornam desagradáveis, com eficiência de trabalho reduzida e aumento no absenteísmo (LIMA; MORORÓ, 2009).

As bibliotecas têm predisposição a apresentar esse problema quando não há um controle sobre a refrigeração e a umidade, além de, às vezes, faltar manutenção preventiva dos aparelhos de ar condicionado, tornando-os disseminadores de micro-organismos e outros poluentes (DANTAS; RICARDI, 2000). A umidade, por exemplo, é um dos fatores determinantes para o crescimento de micro-organismos e que afeta salas mal construídas e pouco arejadas. Tais características põem em risco não somente a preservação do acervo, mas também a garantia do conforto do público e dos trabalhadores que ficam expostos ao ar contaminado (NIELS, 1993).

Para isso, o controle ambiental é de fundamental importância dentro de uma biblioteca, sendo necessária a manutenção da luz, da temperatura e da umidade (KING; PEARSON, 2011; STRAUSZ, 2001), levando a melhorias nas condições de conservação do acervo e da saúde dos funcionários e frequentadores do local. Assim, para controlar todas essas variáveis seria necessário um sistema de refrigeração ambiental de alta qualidade, o que seria inviável em muitos acervos (REISMENEZES, 2009).

No tocante à normatização, em 28 de agosto de 1998 a Portaria 3.523/98 estabelece a aprovação de Regulamento Técnico contendo medidas básicas referentes aos procedimentos de verificação visual e do estado de limpeza, remoção de sujidades por métodos físicos e manutenção do estado de integridade e eficiência de todos os componentes dos sistemas de climatização, para garantir a qualidade do ar de interiores e a prevenção dos riscos à saúde dos ocupantes de ambientes climatizados (DANTAS; RICARDI, 2000), o que representou um grande avanço nacional para diminuir as causas desse problema.

Bem como, foi constatado que ações para prevenir doenças são necessárias, como buscar informação adequada sobre os métodos de desinfecção e conservação; solicitar sugestões de especialistas para a manutenção de um ambiente saudável para o pessoal de serviço e os usuários desse espaço e também do uso de acessórios para proteção dos bibliotecários e pessoas que realizam o serviço de limpeza (STRAUSZ, 2001; COSTA et al. 2008).

Outra normal nacional trata-se da Resolução No 9 de 16 de janeiro de 2003 da ANVISA, que define a orientação técnica sobre a Qualidade do Ar Interior - QAI em ambientes climatizados de uso público e coletivo, considerando o interesse sanitário, segurança, bem-estar e conforto dos ocupantes desses locais. Esta Resolução também tem o objetivo de implementar normas técnicas que visam monitorar a concentração de micro-organismos existentes nos ambientes climatizados, adotando itens de conforto térmico, tais como: temperatura, velocidade do ar, umidade relativa, concentração de CO2 e poeira(BRASIL, 2003), visando trazer melhorias para a saúde do trabalhador.

Entretanto, os funcionários apresentam desconhecimento sobre as consequências da SED, geralmente, os mesmos não se preocupam com a qualidade do ar (MANO 2000), bem como, ser fato, que os sintomas dessa síndrome contribuem para a improdutividade no trabalho, já que os funcionários ficam debilitados para realizar suas atividades (DEGOBBI; GAMBALE) 2008, CASTRO, 2007).

Nesse ínterim, a presente pesquisa objetiva analisar o entendimento dos trabalhadores de uma biblioteca pública de referência no munícipio de Fortaleza-CE, sobre a qualidade do ar, visando contribuir para melhorias pontuais no monitoramento e controle ambiental, justificando a pesquisa e permitindo avanços dessa temática no Brasil.

\section{METODOLOGIA}

A presente pesquisa caracteriza-se como observacional descritiva transversal com abordagem qualiquantitativa (GIL, 1994), na qual se realizou aplicação de questionários juntamente a 38 funcionários de uma biblioteca pública de referência do munícipio de Fortaleza, Ceará.

Conex. Ci. e Tecnol. Fortaleza/CE, v. 10, n. 3, p. 118 - 124, nov. 2016 
Durante o período de janeiro a abril/2013 foram elaborados e aplicados questionários contendo 20 questões objetivas (tipo múltipla escolha), categorizadas em perfil do trabalhador, atividade laboral, estado de saúde e qualidade do ar do ambiente de trabalho, cujo conteúdo levou em consideração artigos de referência nacional e internacional (BELLUSCI, 1998, BETTINI, 2006. FLECK et al., 1999; NAHAS et al., 2009; NASCIMENTO, 2011, SILVEIRA, 2001; VALINOTE, 2011), entre outros, em seguida, houve a adequação das questões para o tema qualidade do ar.

Concomitantemente, a cada funcionário participante foi entregue e assinado o termo de consentimento livre e esclarecido, onde ficava assegurado que a pesquisa não traria nenhuma forma de prejuízo, dano ou transtorno para aqueles que participassem, bem como, que todas as informações obtidas pela análise dos questionários seriam mantidas em sigilo e suas identidades não seriam reveladas.

Os funcionários que responderam aos questionários estavam alocados em 9 setores da biblioteca, distribuídos em 3 acervos (acervo geral, acervo infantil e acervo de obras regionais), 2 recepções (recepção aos usuários e recepção aos funcionários), setor administrativo, setor técnico, setor de obras raras e setor de braille). A escolha dos locais citados acima levou em consideração o elevado número de trabalhadores que transitam permanentemente ou ocasionalmente em seus espaços, bem como ao tipo de climatização, se artificial ou natural, a qual o trabalhador está exposto.

Os dados referentes à avaliação dos questionários são apresentados através de frequências simples e relativas e os pontos centrais das discussões envolvidas são debatidos à luz da literatura atual e pertinente sobre a temática.

\section{RESULTADOS E DISCUSSÕES}

Foram analisados 38 funcionários, assim alocados: acervo geral (06 funcionários), acervo infantil (04), acervo de obras regionais (04), recepção aos usuários (04), recepção aos funcionários (04), setor administrativo (05), setor técnico (04), setor de obras raras (04) e setor de braille (03). Em 100\% dos ambientes analisados foi observada a climatização artificial disponível para o trabalhador.

\subsection{PERFIL DO TRABALHADOR}

O perfil do trabalhador avaliado mostrou que a média etária do público analisado foi de 42 anos (23 anos a menor idade e 64 anos a maior idade), constituído de 24 mulheres (63\%) e 14 homens (37\%), 100\% não fumantes, com uma carga horária oscilando de 20 a 40h/semana. Os funcionários detêm uma média de 12 anos de trabalho em seus cargos, sendo 2 meses o menor tempo e 34 anos o maior tempo de dedicação a carreira dentro da biblioteca. Com base nos dados citados acima, constatou-se a possibilidade de analisar um grupo com considerável experiência em suas funções e no ambiente laboral.

\subsection{ATIVIDADE LABORAL}

No tocante à atividade laboral foi questionada a carga horária extra de trabalho, apenas $8 \%$ dos participantes fazem horas extras. Ao serem perguntados sobre a qualidade ambiental dos setores: $55 \%$ e $52 \%$ consideram boas as condições de limpeza e de temperatura/umidade, respectivamente. Ao indagar sobre o uso de Equipamentos de Proteção Individual - EPIs, 52\% responderam que não usam, e, quando solicitado aos participantes classificarem o ar de seu ambiente de trabalho, $89 \%$ rotularam o ar como empoeirado ou com presença de odores.

Os dados sobre a qualidade ambiental dos setores foram medianos, uma vez que, o efeito da temperatura e da umidade no ambiente deve ser destacado, apesar de atualmente encontrar-se disponível tecnologia sofisticada para controle de tais variáveis, o setor de preservação e conservação de acervos necessita de meios para correlacioná-las diretamente com a qualidade do ar das bibliotecas (KING; PEARSON, 2011; STRAUSZ, 2001, REILLY; NISHIMURA; ZINN, 2001). Nesse ínterim, a literatura também reporta que o excesso de umidade apressa a acidificação do papel, enquanto a baixa umidade conduz ao dessecamento, deixando-o quebradiço e frágil. Concomitantemente, essa variação de umidade favorece o desenvolvimento de microorganismos, com destaque para os bioindicadores da qualidade do ar, os fungos(VALLE, 1991, PANTOJA et al. 2012).

Ainda sobre a categoria atividade laboral, ao questionar sobre o uso de EPIs, 52\% responderam que não usam, com destaque para a não utilização de máscaras e luvas, resultados que corroboram a literatura, como observado em estudo anterior (PALETTA; YAMASHITA; PENILHA, 2005), onde afirmam que os profissionais que atuam diretamente nos processos de higienização de documentos em bibliotecas não utilizam os EPIs, podendo vir a acarretar manifestações alérgicas como, dermatites, rinites, irritação ocular e problemas respiratórios. Sabe-se que compete ao empregador adquirir e fornecer os EPIs ao empregado, bem como orientar e treinar, sendo incontestável que as instituições devem analisar o custo-benefício de programas que visam ga- 
rantir a integridade física e segurança dos seus funcionários (COSTA et al. 2008).

Dentro desse contexto, também foi solicitado que os participantes classificassem o ar de seu ambiente de trabalho, $89 \%$ classificaram o ar como empoeirado ou com presença de odores, apenas os funcionários alocados ao setor de obras raras (11\%) classificaram o ar como limpo, bem como, ao serem questionados sobre o quão saudável é o ambiente de trabalho, apenas os $11 \%$ do setor de obras raras classificaram como saudável, os demais, $89 \%$, consideraram muito pouco saudável ou ruim. Tais dados apontam existir a preocupação dos gestores com o setor que armazena obras de considerável valor histórico e cultural, em detrimento aos demais setores, tal atitude não corrobora a literatura. Segundo estudo internacional (FANGER, 2000), a qualidade do ar de interiores mostra ter uma influência significativa e positiva na produtividade de trabalhadores de escritórios, sendo $6,5 \%$ maior a produtividade com boa qualidade do ar. Portanto, há um forte incentivo econômico para melhorar a qualidade do ar de interiores.

\subsection{ESTADO DE SAÚDE}

$\mathrm{Na}$ categoria estado de saúde, apenas 7 funcionários (18\%) não assinalaram queixas, enquanto que $82 \%$ (31 funcionários) apresentaram sinais relacionados à SED, com um total de 7 queixas distintas, destaque para $29 \%$ que se queixaram de garganta seca e irritada, $19 \% \mathrm{com}$ espirros e coceira nasal e $15 \%$ apresentaram olhos lacrimejantes (ver Figura 1 ).

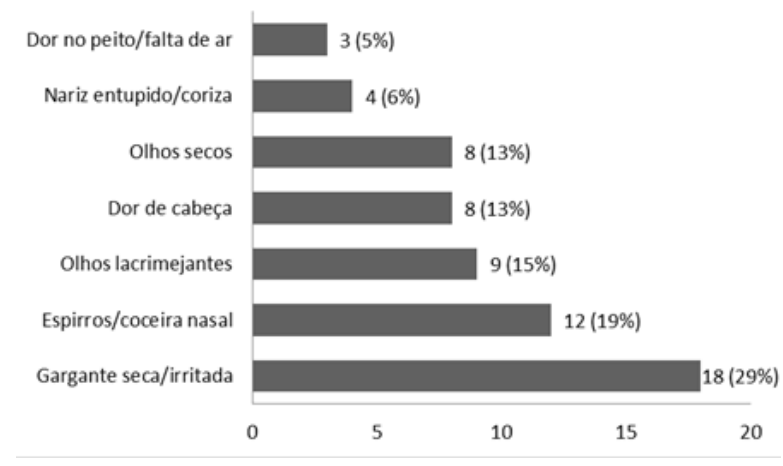

Figura 1: Distribuição das frequências absoluta e relativa de funcionários que apresentaram sintomas relacionados à Síndrome dos Edifícios Doentes, para esse questionamento, o funcionário poderia assinalar mais de uma resposta

De acordo com a Organização Mundial de Saúde, o diagnóstico da SED é eminentemente epidemiológico, logo, na ausência de diagnóstico de patologias definidas, o diagnóstico se faz pela ocorrência de dois ou mais sintomas, que se sucedem pelo menos duas vezes na semana, no interior do prédio, e regridem quando a pessoa se afasta do ambiente em questão (WHO, 2006).

A situação apresentada pelos funcionários analisados no presente artigo assemelha-se ao caso que ocorreu na Biblioteca Central de Manguinhos no Rio de Janeiro durante os anos de 1996 e 1997, a biblioteca em questão sofreu intensa proliferação de fungos, que ocasionavam dores de garganta e de ouvido, alergias respiratórias, sinusite, gripes e pneumonias, devido às baixas temperaturas no ambiente e o tempo de permanência diária de 8h no local. Após uma análise de 122 funcionários através de questionários, $50 \%$ dos trabalhadores se queixaram de problemas dermatológicos e 37,7\% de problemas respiratórios, após a constatação do problema foi criada uma comissão de saúde com o objetivo de monitorar a qualidade do ambiente (STRAUSZ, 2001, STRAUSZ; MACHADO; BRICKUS, 2007).

Quando questionados a respeito das queixas a administração da biblioteca sobre a qualidade do ar do ambiente de trabalho, $71 \%$ nunca se queixaram, dados que vão de encontro à própria legislação brasileira, a qual afirma que o trabalhador deve levar a queixa ao seu superior imediato para a investigação e resolução de qualquer situação de desconforto dentro do ambiente de trabalho (TEIXEIRA 2013). Ao serem inquiridos sobre o quão saudável é o ar de seu ambiente de trabalho, 53\% o consideram muito pouco saudável e $37 \%$ avaliam como ruim sua capacidade respiratória atual. Os resultados apontam a importância de se investir mais na educação, como um instrumento eficaz no processo de conscientização e na luta contínua para tentar diminuir os agravos à saúde do trabalhador (LIMA; MORORÓ, 2009).

Ainda nessa categoria, questionou-se o conhecimento sobre a SED, $100 \%$ nunca ouviram a respeito, dado considerado alarmante, tal falta de informação e desconhecimento sobre a SED e suas consequências fazem os funcionários, geralmente, não se preocuparem com qualidade do ar, devido as causas estarem escondidas em elementos invisíveis e aparentemente inofensivos (MANO 2000), bem como, ser fato, que os sintomas dessa síndrome contribuem para a improdutividade no trabalho, já que os funcionários ficam debilitados para realizar suas atividades (CASTRO, 2007).

Bem como, constata-se que quando maior o tempo de trabalho (em anos) em seus cargos maior o número de queixas, havendo uma maior ocorrência a partir dos 6 anos de prestação de serviço (ver Figura 2). Ao questionar a respeito das queixas à administração da biblioteca sobre a qualidade do ar do ambiente de trabalho, $71 \%$ nunca se queixaram e $100 \%$ dos trabalhadores nunca ouviram a respeito da SED. 


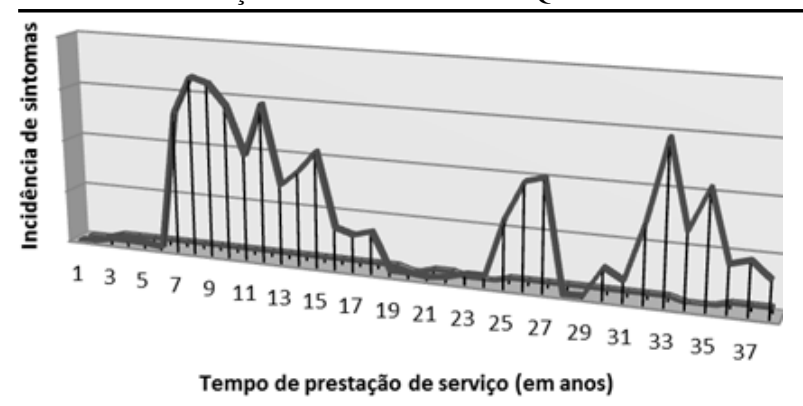

Figura 2: Diagrama de dispersão entre o aparecimento de sintomas e tempo de prestação de serviço em anos, havendo uma maior ocorrência a partir dos 6 anos de carga laboral.

\subsection{QUALIDADE DO AR DO AMBIENTE DE TRABA- LHO}

Frente à categoria qualidade do ar do ambiente de trabalho, constatou-se que $37 \%$ já faltaram mais de 9 dias devido a problemas de saúde nos últimos 12 meses. Bem como, ao indagar sobre a frequência com qual os funcionários fazem de exames de rotina (como exame de sangue, urina, raio $\mathrm{X}$ ), $74 \%$ raramente realizam. Ao rotularem sua saúde apenas $18 \%$ consideram ruim, a maioria $(68 \%)$ classifica como boa, $29 \%$ responderam que após um dia de trabalho apresentam esgotamento físico e mental e, por fim, $45 \%$ não percebem uma possível influência negativa de seu trabalho em sua qualidade de vida.

O percentual apresentado de faltas ao trabalho coadunase com a literatura, já que, segundo alguns pesquisadores, os bioaerossóis de ambientes internos têm sido apontados como uma das principais causas de problemas respiratórios que culminam com a ausência de estudantes à escola e profissionais ao trabalho, ou a baixa produtividade em ambientes ocupacionais (SCHLEIBINGER et al., 2008).

A despeito da imensurável importância das bibliotecas públicas para o patrimônio cultural da humanidade, nem sempre tais instituições dispõem de corpo técnico habilitado para sua correta manutenção, nem dotações orçamentárias compatíveis, sendo necessária a continuidade de estudos correlacionando contaminações ambientais, datações das obras, tipos de climatização do ambiente, analises das percepções ocupacionais, entre outros, sendo fato que muito ainda precisa ser feito frente a qualidade do ar de bibliotecas (PANTOJA et al. 2012).

Os resultados obtidos revelam percentual elevado para a presença de sintomas indicativos da Síndrome dos Edifícios Doentes, bem como a fragilidade ou mesmo ausência de conhecimento dos trabalhadores da biblioteca sobre a referida síndrome, indicando, com base no discernimento dos trabalhadores, a necessidade de implementar normas mínimas no trato com as unidades documentais e o espaço físico das bibliotecas, bem como contribuir com a compilação de dados epidemiológicos da SED que podem repercutir ainda na conservação da saúde de seus usuários.

Todavia, para que sejam construídas políticas de saúde ambientalmente coerentes e eficazes, é necessário dar continuidade com estudos bem delineados sobre a relação entre poluição do ar e saúde do trabalhador, sendo, dessa maneira, possível garantir uma melhor qualidade na informação que será utilizada pelos gestores, contribuindo para o gerenciamento e implementação de novas políticas.

\section{CONSIDERAÇÕES FINAIS}

Por fim, aspira-se que a presente pesquisa, ao demonstrar a fragilidade do conhecimento dos trabalhadores sobre a qualidade do ar, leve a maior divulgação da temática em ambientes ocupacionais, como as bibliotecas, e, como consequência, melhorias pontuais no monitoramento e controle ambiental aéreo, permitindo avanços dessa temática no Brasil.

\section{REFERÊNCIAS BIBLIOGRÁFICAS}

\section{REFERÊNCIAS}

BELLUSCI, S. M. Envelhecimento e condições de trabalho em servidores de uma instituição judiciária: Tribunal Regional Federal da $3^{a}$ Região. Dissertação (Mestrado em Saúde Pública) — Faculdade de Saúde Pública, Universidade de São Paulo, São paulo, 1998. $101 \mathrm{f}$.

BETTINI, D. R. Qualidade do Ar em Laboratório Climatizado de Anatomia Patológica. Avaliação de Agentes Químicos: Faculdade de Ciências Médicas da UERJ. Dissertação (Mestrado em Engenharia Ambiental) - Universidade do Estado do Rio de Janeiro, Rio de Janeiro, 2006. 158 f.

BRASIL. Determina a publicação de Orientação Técnica elaborada por Grupo Técnico Assessor, sobre Padrões Referenciais de Qualidade do Ar Interior, em ambientes climatizados artificialmente de uso público e coletivo. Brasília, 2003. Ministério da Saúde. Agência Nacional de Vigilância Sanitária. Resolução RE n ${ }^{0} 09$, de 16 de janeiro de 2003. Diário Oficial da União da República Federativa do Brasil, Brasília, 20 jan. 2003. 
PERCEPÇÃO OCUPACIONAL: A QUALIDADE DO AR INTERNO EM BIBLIOTECA PÚBLICA, CEARÁ, BRASIL.

CASTRO, K. D. Impactos da qualidade do espaço arquitetônico na produtividade do trabalhador. Pretexto, v. 8, n. 2, p. 23-38, 2007.

COSTA, R. C.; FERNANDES, L. F. O.; LEMOS, A. J. H.; SILVA, R. R. Ocorrência de fungos filamentos em acervo da Faculdade de Medicina da Universidade Federal de Goiás. Revista de Patologia Tropical. v. 37, p. 65-69, 2008.

DANTAS, E. H. M.; RICARDI, E. Qualidade Total. Revista Proteção. v. 98, n. 30, p.40-43, 2000.

DEGOBBI, C. M.; GAMBALE, W. Síndrome dos Edifícios Doentes. Microbiologia in foco. v. 4, n.2, p. 19-32, 2008.

FANGER, P. O. Indoor air quality in the $21^{\text {st }}$ century: Search for excellence. Indoor Air. v. 10, p. 68-73, 2000.

FLECK, M. P. A.; LEAL, O. F.; LOUZADA, S.; XAVIER, M.; CHACHAMOVICK, E.; VIEIRA, G.; SANTOS, L.; PINZON, V. Desenvolvimento da versão em português do instrumento de avaliação de qualidade de vida da OMS (WHOQOL-100). Revista Brasileira de Psiquiatria. v. 21, n. 1, p. 19-27, 1999.

GIL, A. C. Métodos e técnicas de pesquisa social. São Paulo: Atlas, 1994.

HUSMAN, T.; MEKLIN, T.; VEPSALAINEN, A.; VAHTERISTO, M.; KOIVISTO, J.; HALLA-AHO, J.; HYVARINEN, A.; KOPONEN, V.; NEVALAINEN, A. Respiratory infections among children in moisture damaged schools. In: 9th International Conference on Indoor Air Quality and Climate. Proceedings. Santa Cruz, California, United States of America: , 2002. v. 2, p. 484-490.

KING, S.; PEARSON, C. Controle ambiental para instituições culturais: planejamento adequado e uso de tecnologias alternativas. Conservação: Conceitos e práticas. Rio de Janeiro: Editora UFRJ, p. 41-64, 2011.

LIMA, A. F. M.; MORORÓ, M. M. S. Avaliação da salubridade em espaços internos climatizados na cidade de Fortaleza, para a promoção de edificações saudáveis. In: V Encontro Nacional e III Encontro Latino-Americano sobre Edificações e Comunidades Sustentáveis. Recife, Pernambuco, Brasil: , 2009. p. 2.

MANO, C. Prédios doentes. Revista Exame. v. 720, 2000 .
MEHTAL, S.; SMITH, K.; BALAKRISHNAN, K.; SANKAR, S.; PADMAVATHI, R.; KUMAR, S.; AKBAR, S. Using household characteristics to predict respirable particulate levels in rural households of andhra pradesh, india. In: 9 th International Conference on Indoor Air Quality and Climate. Proceedings. Santa Cruz, California, United States of America: , 2002. p. 506-601.

NAHAS, M. V.; RABACOW, F. M.; PEREIRA, S. V.; BORGATTO, A. F. Reprodutibilidade de uma escala para avaliar a percepção dos trabalhadores quanto ao ambiente e às condições de trabalho. Revista Brasileira de Saúde Ocupacional, v. 34, n. 120, p. 179-183, 2009.

NASCIMENTO, G. C. Avaliação da qualidade do ar de ambientes internos: biblioteca pública. Dissertação (Mestrado em Engenharia Hidráulica e Saneamento) - Escola de Engenharia de São Carlos, Universidade de São Paulo, São Paulo, 2011.

NIELS, M. Alergia: um texto ilustrado. Rio de Janeiro: Revinter, 1993. 481p.

PALETTA, F. A. C.; YAMASHITA, M. M.; PENILHA, D. F. Equipamentos de proteção individual (EPIs) para profissionais de bibliotecas, centros de documentação e arquivos. Revista Digital de Biblioteconomia e Ciência da Informação, v. 2, n. 2, p. 67-79, 2005.

PANTOJA, L. D. M.; RIZZO, R. S.; CARVALHO, B. S.; FERREIRA, V. C.; GALAS, K. S.; FONSECA, F. R. M.; PAIXÃO, G. C. Constituição da micobiota aérea de bibliotecas públicas no município de Fortaleza, Estado do Ceará, Brasil. Encontros Bibli: revista eletrônica de biblioteconomia e ciência da informação, v. 17, n. 34, p. 31-41, 2012.

REILLY, J. M.; NISHIMURA, D. W.; ZINN, E. Novas ferramentas para preservação: avaliando os efeitos ambientais a longo prazo sobre coleções de bibliotecas e arquivos. Rio de Janeiro: Projeto Conservação Preventiva em Bibliotecas e Arquivos, 2001.

REIS-MENEZES, A. A. Fungos em Bibliotecas: Frequência dos gêneros em livros e elaboração de teste para avaliação da biorreceptividade em papéis. Tese (Doutorado em Microbiologia) — Instituto de Biomédicas, Universidade de São Paulo, São Paulo, 2009. $90 \mathrm{f}$.

ROBERTSON, G. Sick Buildings: Effects, causes, analysis and prevention. In: Councill on tall buidings and urban habitat, Bethlehem, Pennsylvania, United Stated of America, p. 70-88, 1995. Rehabilitation of Damaged Buildings. 
ROCHA, L. S. Relatório final das atividades do projeto biblioteca de Manguinhos e a Síndrome do Edifício Doente. Rio de Janeiro, 2008. 156 p. CNPQ, Coordenação do Programa Rhae.

SAUNDERS, T. Sua saúde e o ambiente que construímos: a síndrome do sapo cozido. São Paulo: Editora Cultrix, 2005.

SCHLEIBINGER, H.; LAUSSMANN, D.; BORNEHAG, C.-G.; EIS, D.; RUEDEN, H. Microbial volatile organic compounds in the air of moldy and mold-free indoor environments. Indoor Air, v. 18, n. 1, p. 113-124, 2008.

SILVEIRA, M. G. Avaliaçäo da qualidade do ar em um aeroporto na cidade do Rio de Janeiro. Tese (Doutorado em Ciências) - Fundação Oswaldo Cruz, Escola Nacional de Saúde Pública, Centro de Estudos de Saúde do Trabalhador e Ecologia Humana, Manguinhos, Rio de Janeiro, 2001. 153 f.

STRAUSZ, M. C. Análise de um acidente fúngico na Biblioteca Central de Manguinhos: um caso de síndrome do edifício doente. Dissertação (Mestrado em Saúde Pública) — Fundação Oswaldo Cruz, Escola Nacional de Saúde Pública, Manguinhos, Rio de Janeiro, 2001.

STRAUSZ, M. C.; MACHADO, J. M. H.; BRICKUS, L. d. S. R. Análise de um acidente por contaminação fúngica em uma biblioteca pública no município do Rio de Janeiro. Revista Brasileira de Saúde Ocupacional, v. 32, n. 115, p. 1-9, 2007.

TEIXEIRA, J. C. A legislação de saúde do trabalhador aplicável e vigente no Brasil. Disponível em: <http://www.pgt.mpt.gov.br>, Acesso em: 30 nov, 2013.

VALINOTE, H. C. Ambiente de trabalho e qualidade de vida dos trabalhadores da construção civil de uma construtora de Goiânia. Dissertação (Mestrado em Ciências Ambientais e saúde) - Programa de Pós-Graduação em Ciências Ambientais e Saúde, Pontifícia Universidade Católica de Goiás, Goiânia, 2011. $128 \mathrm{f}$.

VALLE, C. A. Subsídios para uma política de preservação e conservação de acervos em bibliotecas universitárias brasileiras. Dissertação (Mestrado em Biblioteconomia e Documentação) — Universidade de Brasília, Brasília, 1991. 106 f.

WHO. World Health Organization. Environment and Air. New York/EUA, 2006. Portal eletrônico.
Apresenta informações sobre a saúde mundial. Disponível em: <http://www.who.int $>$. Acesso em: 25 nov. 2013. 